\title{
FRACTION OF POSITRONIUM FORMATION AT SEMICONDUCTOR SURFACE
}

\author{
S.B. Shrivastava AND A. Upadhyay \\ School of Studies in Physics, Vikram University, Ujjain (M.P.), 456010 India \\ (Received May 4, 1998; revised version December 8, 1998; \\ in final form March 30, 1999)
}

The fraction of positronium formation $\left(f_{\mathrm{ps}}\right)$ has been calculated in $\mathrm{Ge}(110), \mathrm{Ge}(111), \mathrm{Si}(110)$ and $\mathrm{Si}(111)$ surfaces by solving the diffusion equation for positrons in semiconductors and by setting up the rate equation to describe the processes that are supposed to occur when a thermalised positron encounters the surface including the trapping of positrons in neutral and negative vacancies. Certain parameters used in the evaluation of $f_{\mathrm{ps}}$, e.g., the bulk annihilation rate $\left(\lambda_{s}\right)$, the positron diffusion length $\left(L_{+}\right)$, the diffusion coefficient $\left(D_{+}\right)$and the implantation profile parameter $(A)$, have been taken from the experiments. The calculated values of $f_{\mathrm{ps}}$ as a function of incident positron energy and temperature in $\mathrm{Ge}(110)$ and $\mathrm{Si}(111)$ have been compared with the experimental results. It has been found that in general the calculated results are in good agreement with the experimental ones. The calculation also confirms that the trapping rate of positrons into negative vacancy has a $T^{-1 / 2}$ dependence with respect to the temperature.

PACS numbers: 78.70.Bj, 71.60.+z, 68.35.Fx

\section{Introduction}

The recent development of variable energy positron beams for study of solid material has resulted in a new technique that is capable of probing surfaces as well as near surface phenomena [1]. More recently, there has been a growing interest in the investigation of near surface defects in the case of semiconductors using the above technique $[2,3]$. The first few studies were bulk Doppler broadening measurements of the positron mobility in solid state $\mathrm{Ge}$ and Si by Mills and Pheiffer [4] yielding values for positron diffusion coefficients $\left(D_{+}\right)$that were comparable with other solids. These measurements were followed by positron beam studies of $D_{+}$vs. temperature in Ge by Jorch et al. [5], measuring the fraction of positronium formation. In this work, it was observed that $D_{+}$did not follow any simple model. Another detailed study of positron diffusion was carried out for several Si crystals by Nielsen et al. [6]. Lynn et al. [7] demonstrated the use of variable 
energy positron beam technique for the investigation of near surface defects by measuring the positron line shape parameter in $\mathrm{Si}$ and $\mathrm{SiO}_{2}$. The electronic structure of vacancy type defects in semiconductors is much more complicated than that in metals. Positron trapping has been much less studied in semiconductors than in metals where the subject is well documented both theoretically and experimentally [8]. In semiconductors there is experimental evidence $[9,10]$ that the characteristics of positron trapping into vacancy defects vary considerably between different semiconductors.

Earlier [11] we had discussed the mechanism of positron annihilation at metal surfaces, by considering the positron diffusion and trapping of positrons into surface states and near surface defects. The model was applied to obtain the fraction of positronium formation and positron lifetime in certain metal surfaces e.g., $\mathrm{Al}$ and $\mathrm{Ag}$, etc. In the present work, we use a similar approach but consider the case of slow positrons incident on the surface of a semiconductor. The aim is to understand the positron diffusion and trapping of positrons in near surface defects. The sensitivity of slow positrons to surface condition arises from the fact that after thermalization a fraction of implanted positrons will diffuse back to the sample surface where it may be trapped in to near surface defects. The diffusion of positrons is described by solving the one-dimensional diffusion equation for positrons in semiconductors. The rate equations are then set up to describe the various processes that are supposed to occur when a thermalized positron encounters the surface. These are then used to obtain the fraction of positronium formation as a function of incident energy of positrons and of temperature.

\section{Calculation of positronium fraction}

The three-dimensional diffusion equation is given by

$$
\frac{\partial u(r, t)}{\partial t}=D_{+} \nabla^{2} u(r, t)-\lambda_{\mathrm{eff}} u(r, t)-\frac{\partial}{\partial r}\left[v_{\mathrm{d}} u(r, t)\right],
$$

where $D_{+}$is the positron diffusion coefficient, $u(r, t)$ is the positron density as a function of both time and position, $\lambda_{\text {eff }}$ is the effective annihilation rate of the positrons in a truly diffusing state and $v_{\mathrm{d}}$ is the field dependent drift velocity. We describe the motion of positrons implanted in the semiinfinite medium with a given implantation profile using the one-dimensional diffusion equation:

$$
D_{+} \frac{\partial^{2} u(x, t)}{\partial x^{2}}-\frac{\partial}{\partial x}\left[v_{d}(x, t)\right]-\lambda_{\mathrm{eff}} u(x, t)=\frac{\partial u(x, t)}{\partial t} .
$$

The diffusion equation is solved, subject to the boundary condition $u(0, t)=0$ (absorbing boundary) and $u(x, 0)=C_{0}(x)$ implantation profile. Considering the exponential type of implantation profile

$$
C_{0}(x)=\frac{1}{a} \exp \left(-\frac{x}{a}\right)
$$

and $a=A E^{n}$, where $E[\mathrm{keV}]$ is the energy of the incident positrons. The value of $n$ is taken to be 1.6 as per experimental observations [5]. The solution of Eq. (2) so obtained is given by 


$$
\begin{aligned}
& u(x, t)=\sum_{n} A_{n}^{\prime} \exp \left[-\left(-\frac{n^{2} \pi^{2}}{L_{+}^{2}}+\frac{v_{\mathrm{d}}^{2}}{4 D_{+}^{2}}\right) D_{+} t-\lambda_{\mathrm{eff}}\right] \\
& \times \sin \left( \pm \frac{n \pi}{L_{+}}\right) x \exp \left[\left(v_{\mathrm{d}} / 2 D_{+}\right) x\right]
\end{aligned}
$$

where

$$
A_{n}^{\prime}=\sqrt{\frac{2}{a} \frac{\left(n \pi / L_{+}\right)}{\left(1+v_{\mathrm{d}} a / 2 D_{+}\right)}}
$$

and $L_{+}$is the diffusion length.

The desired rate of positrons reaching the surface is

$$
N(t)=\left.D_{+} \frac{\partial u(x, t)}{\partial x}\right|_{x=0} \text {. }
$$

Thus we get

$$
N(t)=\sum B_{n} \exp \left(-b_{n} t\right)
$$

here

$$
B_{n}=D_{+}\left(\frac{n \pi}{L_{+}}\right)^{3 / 2} \sqrt{\frac{2}{a\left(1+a v_{\mathrm{d}} / 2 D_{+}\right)}}
$$

and

$$
b_{n}=\left[\left(-\frac{n^{2} \pi^{2}}{L_{+}^{2}}+\frac{v_{\mathrm{d}}^{2}}{4 D_{+}^{2}}\right) D_{+}+\lambda_{\mathrm{eff}}\right] .
$$

At the surface the positrons may enter one of the three main channels with roughly equal probabilities: (i) emission as free positrons, (ii) emission as free Ps or (iii) localization within a surface state or in the near surface defects. Puska et al. [12] have done a systematic theoretical survey and discussed the trapping of positrons into vacancy type defects in semiconductors. Defects in semiconductors may exist in several charge states. Positive defects are expected to repeal positrons, negatively charge defects are expected to be strongly attractive to positrons due to their attractive long range Coulomb potential. This Coulomb tail can affect the capture mechanism into vacancies by inducing several positron charged states with low binding energy. In the present work, first we consider a positron trapping process in which a delocalized positron is trapped into the ground state, at a neutral vacancy or in a shallow defect. Secondly, a delocalised positron is trapped into a Rydberg state at a negative vacancy or ion. The rate equations to describe these processes are written as follows:

Let $n_{\mathrm{B}}$ be the fraction of positrons in the bulk state at the given instant of time. These may be annihilated inside the solid by an annihilation rate $\lambda_{B}$, or transferred to the surface by a back diffusion rate $N(t)$. We, thus, write for annihilation,

$$
\frac{\partial n_{\mathrm{B}}(t)}{\partial t}=-\lambda_{\mathrm{B}} n_{\mathrm{B}}(t)-N(t)
$$


The other equations could be written in an analogous manner:

or

$$
\frac{\partial n_{\mathrm{S}}(t)}{\partial t}=-\left(\alpha_{e^{+}}+\alpha_{\mathrm{ST}}+\alpha_{\mathrm{SO}}+\alpha_{\mathrm{SP}}+\alpha_{\mathrm{SV}-}\right) n_{\mathrm{S}} t+N(t)
$$

$$
\frac{\partial n_{\mathrm{S}}(t)}{\partial t}=-\alpha_{\mathrm{S}} n_{\mathrm{S}} t+N(t)
$$

where $\alpha_{\mathrm{S}}=\alpha_{e^{+}}+\alpha_{\mathrm{ST}}+\alpha_{\mathrm{SO}}+\alpha_{\mathrm{SP}}+\alpha_{\mathrm{SV}-}$,

or

$$
\frac{\partial n_{\mathrm{ST}}(t)}{\partial t}=-\left(\lambda_{\mathrm{C}}+\lambda_{i}+\alpha_{\mathrm{TP}}\right) n_{\mathrm{ST}}(t)+\alpha_{\mathrm{ST}} n_{\mathrm{S}}(t)
$$

$$
\frac{\partial n_{\mathrm{ST}}(t)}{\partial t}=-\alpha_{\mathrm{T}} n_{\mathrm{ST}}(t)+\alpha_{\mathrm{ST}} n_{\mathrm{S}}(t)
$$

where $\alpha_{\mathrm{T}}=\lambda_{\mathrm{C}}+\lambda_{i}+\alpha_{\mathrm{TP}}$,

$$
\begin{aligned}
& \frac{\partial n_{\mathrm{TP}}(t)}{\partial t}=-\lambda_{\mathrm{P}} n_{\mathrm{TP}}(t)+\alpha_{\mathrm{TP}} n_{\mathrm{TP}}(t) \\
& \frac{\partial n_{\mathrm{SV}-}(t)}{\partial t}=-\left(\lambda_{\mathrm{V}}+\alpha_{\mathrm{V}-\mathrm{P}}\right) n_{\mathrm{SV}-}(t)+\alpha_{\mathrm{SV}-} n_{\mathrm{S}}(t)
\end{aligned}
$$

or

$$
\frac{\partial n_{\mathrm{SV}-}(t)}{\partial t}=-\alpha_{\mathrm{V}} n_{\mathrm{SV}-}(t)+\alpha_{\mathrm{SV}-} n_{\mathrm{S}}(t)
$$

where $\alpha_{\mathrm{V}}=\lambda_{\mathrm{V}}+\alpha_{\mathrm{V}-\mathrm{P}}$,

$$
\begin{aligned}
& \frac{\partial n_{\mathrm{V}-\mathrm{P}}(t)}{\partial t}=-\lambda_{\mathrm{P}} n_{\mathrm{V}-\mathrm{P}}(t)+\alpha_{\mathrm{V}-\mathrm{P}} n_{\mathrm{SV}-}(t), \\
& \frac{\partial n_{\mathrm{SO}}(t)}{\partial t}=-\lambda_{\mathrm{O}} n_{\mathrm{SO}}(t)+\alpha_{\mathrm{SO}} n_{\mathrm{S}}(t), \\
& \frac{\partial n_{\mathrm{SP}}(t)}{\partial t}=-\lambda_{\mathrm{P}} n_{\mathrm{SP}}(t)+\alpha_{\mathrm{SP}} n_{\mathrm{S}}(t) .
\end{aligned}
$$

In the above equations $n_{\mathrm{S}}, n_{\mathrm{ST}}, n_{\mathrm{SV}}$ - represent the fraction of positrons in the delocalized surface state, trapped into other surface states including neutral vacancies and shallow defects and trapped into negative ion vacancies, respectively. The $n_{\text {TP }}$, etc. represent the fraction that detrapped from the first subscript state to form positronium, $n_{\mathrm{SO}}$ and $n_{\mathrm{SP}}$ represent the detrapping into ortho and para positronium states. $\alpha_{i j}$ are the transition rates from $i$ th state to $j$ th state and $\lambda_{j}$ are the annihilation rates in the respective states.

Equations (10) to (17) have been solved using appropriate initial conditions and using Eq. (7) for $N(t)$. The fraction of positronium formation at the surface of the semiconductor has been obtained employing the relation

$$
f_{\mathrm{ps}}=\int_{0}^{\infty} \lambda_{\mathrm{P}} n_{\mathrm{TP}}(t) \mathrm{d} t+\int_{0}^{\infty} \lambda_{\mathrm{P}} n_{\mathrm{SP}}(t) \mathrm{d} t \int_{0}^{\infty} \lambda_{\mathrm{P}} n_{\mathrm{V}-\mathrm{P}}(t) \mathrm{d} t .
$$

Putting the value of solutions of Eqs. (10) to (17) and evaluating the integrals one obtains

$$
f_{\mathrm{ps}}=\sum_{n=1}^{\infty} \frac{B_{n}}{b_{n}} \frac{1}{\alpha_{\mathrm{S}}}\left[\frac{\alpha_{\mathrm{SV}-} \alpha_{\mathrm{V}-\mathrm{P}}}{\alpha_{\mathrm{V}}}+\frac{\alpha_{\mathrm{ST}} \alpha_{\mathrm{TP}}}{\alpha_{\mathrm{T}}}+\alpha_{\mathrm{SP}}\right],
$$


where $\alpha_{\mathrm{S}}=\alpha_{e+}+\alpha_{\mathrm{ST}}+\alpha_{\mathrm{SO}}+\alpha_{\mathrm{SP}}+\alpha_{\mathrm{SV}-}$. Equation (19) can be used to calculate the fraction of positronium formation in semiconductors.

Puska et al. [12] have shown that the trapping coefficient for neutral vacancy shows an increasing trend as temperature rises. Further, the trapping rate is expected to be proportional to the thermal vacancy concentration. We, therefore, take the trapping rate of positron into neutral vacancies and shallow defects as

$$
\alpha_{\mathrm{ST}}=A_{1} \exp \left(-\frac{S}{k_{\mathrm{B}}}-\frac{H_{\mathrm{IV}}}{k_{\mathrm{B}} T}\right)
$$

where $S$ is the thermal entropy and $H_{\text {IV }}$ neutral vacancy formation enthalpy. The value of $H_{\mathrm{IV}}$ derived following Ref. [13] from the relation $H_{\mathrm{IV}}=10 k_{\mathrm{B}} T_{\mathrm{m}}$, where $T_{\mathrm{m}}$ is the melting temperature and $k_{\mathrm{B}}$ is Boltzmann constant. Puska et al. [12] also studied the positron trapping coefficient for negative vacancies. They obtained the initial and final state using the potential with the attractive. Coulomb tail. The trapping coefficient for negative vacancies at low temperature was found to be proportional to $T^{-1 / 2}$. For the doubly negative vacancy also the trapping coefficient was found to diverge as $T^{-1 / 2}$. We have, thus, taken for the trapping rate

$$
\alpha_{\mathrm{SV}-}=A_{2} T^{-1 / 2}
$$

where $A_{2}$ is a constant.

To obtain the detrapping rate $\alpha_{\text {TP }}$ we observe following Ref. [12] that when the delocalised and the trapping states are in thermal equilibrium the detrapping rate at a given temperature $T$ may be described by the relation

$$
\alpha_{\mathrm{TP}}=A_{3} T^{3 / 2} \exp \left(-\frac{E_{\mathrm{b}}}{k_{\mathrm{B}} T}\right)
$$

where $E_{\mathrm{b}}$ is the positron binding energy in shallow traps. The above relation (Eq. (22)) was also used to calculate $\alpha_{\mathrm{V}-\mathrm{p}}$, the detrapping rate corresponding to negative vacancy.

In Eqs. (8) and (9) the second term is evaluated as follows: Using Einstein relation the mobility $\mu$ is related to the diffusion coefficient by $\mu=D_{+} / k_{\mathrm{B}} T$. But $\mu=v_{\mathrm{d}} / \varepsilon e, \varepsilon$ being the electric field and $e$ is the positron charge. Giving

$$
v_{\mathrm{d}}=\frac{\varepsilon e D_{+}}{k_{\mathrm{B}} T}
$$

Lynn et al. [7] suggested a relationship between the diffusion length $L_{+}$and electric field $\varepsilon$ in $\mathrm{Si}$, thus $L_{+}=k_{\mathrm{B}} T / \varepsilon e$. The temperature dependence of the diffusion coefficient has been discussed by Simpson et al. [14] in Si. They have suggested that $D_{+}$is proportional to $T^{-1 / 2}$. We have thus, taken $D_{+}=C_{1} T^{-1 / 2}$ incorporating Eqs. (23) one obtains

$$
\frac{v_{\mathrm{d}}^{2}}{4 D_{+}}=\frac{C_{1} T^{-1 / 2}}{4 L_{+}^{2}}
$$




\section{Results and discussion}

Employing the procedure as described above, the fraction of positronium formation has been calculated using Eq. (19) and incorporating Eqs. (8), (9) and (20) to (24). The various parameters applied in the evaluation have been taken mostly from the experimental values. Several constants have been estimated to give good results. All these parameters are listed in Table.

TABLE

Values of different parameters used in the calculation of $f_{\mathrm{ps}}$ alongwith the references from which they are taken.

\begin{tabular}{l|c|c|c|r}
\hline \multicolumn{1}{c|}{ Parameter } & $\mathrm{Ge}(110)$ & Ref. & Si(111) & Ref. \\
\hline$A\left[\mathrm{keV} \mathrm{cm}^{-1}\right]$ & $8.9 \times 10^{-10}$ & {$[5]$} & $2.06 \times 10^{-9}$ & {$[5]$} \\
$\alpha_{\mathrm{SP}}\left[\mathrm{s}^{-1}\right]$ & $2 \times 10^{14}$ & {$[20]$} & $2 \times 10^{14}$ & {$[20]$} \\
$\alpha_{\mathrm{SO}}\left[\mathrm{s}^{-1}\right]$ & $6 \times 10^{14}$ & {$[20]$} & $1 \times 10^{14}$ & {$[20]$} \\
$\lambda_{\mathrm{B}}\left[\mathrm{s}^{-1}\right]$ & $4.27 \times 10^{9}$ & {$[17]$} & $4.6 \times 10^{9}$ & {$[17]$} \\
$\lambda_{\mathrm{S}}\left[\mathrm{s}^{-1}\right]$ & $0.228 \times 10^{9}$ & {$\left[{ }^{*}\right]$} & $2 \times 10^{9}$ & {$\left[{ }^{*}\right]$} \\
$\lambda_{\mathrm{C}}\left[\mathrm{s}^{-1}\right]$ & $1.5 \times 10^{9}$ & {$[19]$} & $0.1 \times 10^{9}$ & {$[17]$} \\
$\lambda_{i}\left[\mathrm{~s}^{-1}\right]$ & $4.47 \times 10^{9}$ & {$[19]$} & $4.47 \times 10^{9}$ & {$\left[{ }^{*}\right]$} \\
$P$ & 0.87 & {$[19]$} & 0.95 & {$\left[{ }^{*}\right]$} \\
$D_{+}\left[\mathrm{cm}^{2} \mathrm{~s}^{-1}\right]$ & 0.2 & {$[1]$} & 2.7 & {$[1]$} \\
$L_{+}[\AA]$ & 1500 & {$[5]$} & 1400 & {$[18]$} \\
$E_{\mathrm{b}}[\mathrm{eV}]$ & $0.94 \times 10^{-3}$ & {$[12]$} & $0.94 \times 10^{-3}$ & {$[12]$} \\
$H_{\mathrm{IV}}[\mathrm{eV}]$ & $1.06 \times 10^{-3}$ & {$\left[{ }^{*}\right]$} & $1.04 \times 10^{-3}$ & {$\left[{ }^{*}\right]$} \\
$A_{1}\left[\mathrm{~s}^{-1} \mathrm{~K}^{-1}\right]$ & $6 \times 10^{11}$ & {$\left[{ }^{*}\right]$} & $1 \times 10^{15}$ & {$\left[{ }^{*}\right]$} \\
$A_{2}\left[\mathrm{~s}^{-1} \mathrm{~K}^{-1}\right]$ & $5 \times 10^{15}$ & {$\left[{ }^{*}\right]$} & $2.5 \times 10^{15}$ & {$\left[{ }^{*}\right]$} \\
$A_{3}\left[\mathrm{~s}^{-1} \mathrm{~K}^{-1}\right]$ & $1.6 \times 10^{10}$ & {$\left[{ }^{*}\right]$} & $1 \times 10^{14}$ & {$\left[{ }^{*}\right]$} \\
$\beta$ & $6.8 \times 10^{-1}$ & {$\left[{ }^{*}\right]$} & $7.9 \times 10^{-1}$ & {$\left[{ }^{*}\right]$} \\
\hline${ }^{*}$ present work
\end{tabular}

The calculated results of positronium fraction $\left(f_{\mathrm{ps}}\right)$ are plotted in Figs. (1) to (4). Figure 1 shows a plot of calculated results of Ps fraction $\left(f_{\mathrm{ps}}\right)$ as a function of incident energy of positron for two temperatures in the case of $\mathrm{Ge}(110)$. The calculated results are compared with the experimental observations of Jorch et al. [5]. One can see from these graphs that the general trends of the experimental curves are well reproduced. $f_{\mathrm{ps}}$ decreases with the increase in incident energy similar to the case of metals. This is due to the fact that with the increase in incident energy the positron annihilation in bulk dominates, thus, reducing the Ps formation at surfaces. It is known that Ps formation does not take place in bulk semiconductors.

In Fig. 2 we have plotted the variation of $f_{\mathrm{ps}}$ with respect to temperature for different values of energy. These results could not be compared with the exper- 
Fig. 1

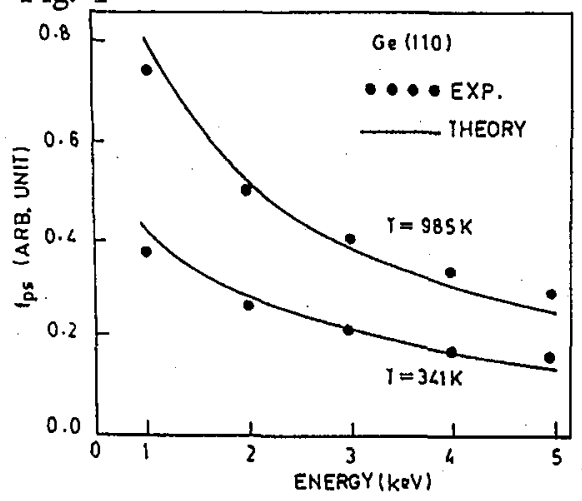

Fig. 3

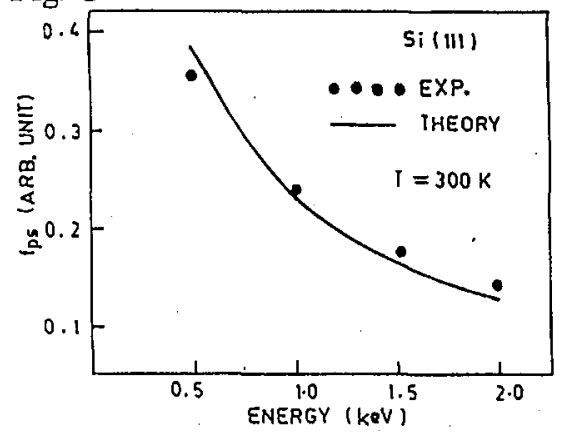

Fig. 2

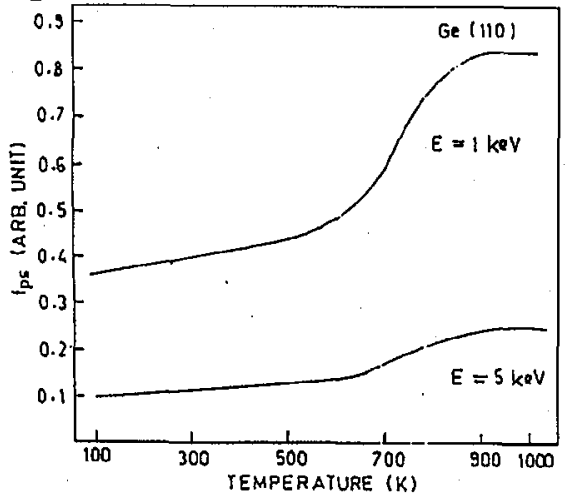

Fig. 4

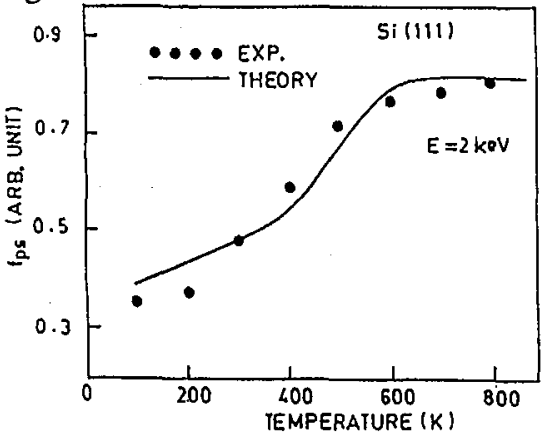

Fig. 1. Comparison of the calculated values of fraction of positronium formation vs. incident energy with the experimental results of Jorch et al. [5] in $\mathrm{Ge}(110)$.

Fig. 2. Variation of the calculated values of fraction of positronium formation with temperature in $\mathrm{Ge}(110)$.

Fig. 3. Comparison of the calculated values of $f_{\mathrm{ps}}$ in $\mathrm{Si}(111)$ with the experimental results of Mills [15] as a function of incident energy.

Fig. 4. Comparison of the calculated values of $f_{\mathrm{ps}}$ with the experimental results of Ref. [16] as a function of temperature in $\mathrm{Si}(111)$.

iments as the latter are not available. The graph shows that with the increase in temperature $f_{\mathrm{ps}}$ increases. The increase is larger in the case of low energy positrons. This is due to the reason that the detrapping rate from the surface states including near surface defects to Ps states increases with temperature.

In Figs. 3 and 4 we have compared the calculated values of $f_{\mathrm{ps}}$ in the case of $\mathrm{Si}(111)$ with the experimental results. In Fig. 3, the positronium fraction is plotted as a function of energy. The experimental points are given by dots and are taken from Ref. [15]. The two results show very good agreement. In Fig. 4 the variation of $f_{\mathrm{ps}}$ in $\mathrm{Si}(111)$ is shown as a function of temperature. The results are also compared with the experimental values of Ref. [16] The graph shows that $f_{\mathrm{ps}}$ first increases and then becomes saturated at high temperatures. 
We have, thus, found that the above model is able to describe the experimental results of slow positron annihilation in semiconductors up to a good degree of extent. In the above description we have invoked the trapping of positrons into near surface defects. This includes the trapping into neutral and negative vacancies. The trapping rates are governed by Eqs. (20) and (21) and detrapping is described by Eq. (22). This confirms that in the case of neutral vacancies the trapping rate has a linear dependence with respect to temperature. While in the case of the negative vacancies it varies with $T^{-1 / 2}$. The above work, it is hoped, will help in the study of near surface defects in semiconductors. More theoretical and experimental efforts are, however, needed to confirm this.

\section{References}

[1] P.J. Schultz, K.G. Lynn, Rev. Mod. Phys. 60, 701 (1988).

[2] R.S. Brusa, M. Duarte Naina, A. Zecca, C. Nobili, G. Ottaviani, R. Tonini, A. Dupasquier, Phys. Rev. B 49, 7271 (1994).

[3] R. Krause-Rehberg, H.S. Leipner, A. Kupsch, A. Polity, Th. Drost, Phys.Rev. B 49,2385 (1994).

[4] A.P. Mills, L. Pheiffer, Phys. Rev. Lett. 36, 389 (1976).

[5] H.H. Jorch, K.G. Lynn, T. McMullen, Phys. Rev. B 30, 93 (1984).

[6] B. Nielsen, K.G. Lynn, A. Vehanen, P.J. Schultz, Phys. Rev. B 32, 2296 (1985).

[7] K.G. Lynn, D.O. Welch, J. Throwe, B. Nielsen, Int. Mater. Rev. 36, 1 (1991).

[8] R.M. Nieminen, M. Manninen, in: Positron in Solids, Ed. P. Hautojarvi, Springer-Verlag, Heidelberg 1979, p. 145.

[9] R.M. Nieminen, in: Positron Solid State Physics, Eds. W. Brandt, A. Dupasquier, North Holland Pub. Co., Amsterdam 1983, p. 359.

[10] S. Dannefaer, S: Kupca, B.G. Hogg, J.P. Kerr, Phys. Rev. B 22, 6135 (1980).

[11] S.B. Shrivastava, V.K. Gupta, Surf. Sci. Letts. 239, L-498 (1990).

[12] M.J. Puska, C. Corbel, R.M. Nieminen, Phys. Rev. B 41, 9980 (1990).

[13] K. Maier, in: Positron Solid State Physics, Eds. W.Brandt, A. Dupasquier, North Holland Pub. Co., Amsterdam 1983, p. 265.

[14] R.I. Simpson, M.J. Stewart, C.D. Beling, M. Charlton, in: Positron Annihilation, Eds. L. Dorikens Vanpraet, M. Dorikens, D. Segers, ICPA VIII, World. Sc. Publ., Singapore 1988, p. 696.

[15] A.P. Mills, Jr., Solid State Commun. 41, 1828 (1978).

[16] A.P. Mills, Jr., Solid State Commun. 31, 623 (1979).

[17] M.J. Puska, in: Positron Annihilation, Eds. L. Dorikens Vanpraet, M. Dorikens, D. Segers, ICPA VIII, World. Sc. Publ., Singapore 1988, p. 101.

[18] J.A. Baker, P.J. Coleman, B. Wakefield, C.J. Gibbings, C.J. Tuppen, Semicond. Sci. Technol. 4, 815 (1989).

[19] S.B. Shrivastava, V.K. Gupta, Acta Phys. Pol. A 79, 6 (1991).

[20] R.V. Levine, L.M. Sander, Solid State Commun. 42, 5 (1982). 